\title{
Estilo de vida de universitários usuários de substâncias nocivas e associação com atividade física do cotidiano
}

\author{
Lifestyle of university students using harmful substances and association with daily physical \\ activity
}

Estilo de vida de los estudiantes universitarios que consumen sustancias nocivas y asociación con la actividad física diaria

Recebido: 01/03/2021 | Revisado: 09/03/2021 | Aceito: 10/03/2021 | Publicado: 18/03/2021

\author{
Higor Santos Fonseca \\ ORCID: https://orcid.org/0000-0003-2676-6576 \\ Universidade Estadual do Paraná, Brasil \\ E-mail: higofonnse@gmail.com \\ Sérgio Roberto Adriano Prati \\ ORCID: https://orcid.org/0000-0003-4375-2467 \\ Universidade Estadual do Paraná, Brasil \\ E-mail: srap@bol.com.br
}

\begin{abstract}
Resumo
O objetivo deste estudo foi analisar o estilo de vida (EV) de universitários usuários de substâncias nocivas e associá-la com a atividade física do cotidiano. Para isso, foram investigados 1405 universitários de um campus universitário de Paranavaí-PR, no qual 330 (23,4\%) foram identificados como usuários de tabaco e/ou drogas (TD) e 401 (28,5\%) de álcool (AL). Além disso, cerca de $85 \%$ dos universitários não tinham hábitos considerados saudáveis de atividades físicas (AF). O EV foi mensurado por meio do questionário "Estilo de Vida Fantástico". Sendo assim, observou-se que o EV dos universitários usuários de substâncias nocivas, em média, foi considerado inadequado $(\mathrm{TD}=58,7 \pm 10,9$; $\mathrm{AL}=59,6 \pm 11,0$, adequado quando $\mathrm{EV} \geq 70 \mathrm{pts}$ ) independentemente do sexo. Quando a AF era adequada (em 14,4\% dos casos), 9,6\% eram usuários de TD e $20 \%$ de AL, já, quando AF era inadequada os níveis de usuários praticamente não se alteravam percentualmente $(\mathrm{TD}=10,5 \%$ e $\mathrm{AL}=20,4 \%)$. Desta forma, conclui-se que o $\mathrm{EV}$ de universitários não era adequado para saúde, além disso, não se observou interferência da prática regular e adequada de atividades física em relação ao uso de substâncias nocivas.
\end{abstract}

Palavras-chave: Estilo de vida; Substâncias nocivas; Atividade física.

\begin{abstract}
The aim of this study was to analyze the lifestyle (LS) of university students who use hazardous substances and associate it with daily physical activity. For this, 1405 university students from the State University of Paraná campus of Paranavaí in Paraná were investigated in which 330 (23.4\%) were identified as tobacco and/or drug users (TD) and $401(28.5 \%)$ of alcohol (AL). In addition, about $85 \%$ of university students did not have healthy physical activity (PA) habits. The LS was measured using the "Fantastic Lifestyle" questionnaire. Thus, it was observed that the LS of university students using harmful substances, on average, was considered inadequate $(\mathrm{TD}=58.7 \pm 10.9 ; \mathrm{AL}=59.6 \pm 11.0$, adequate when LS $\geq 70$ pts) regardless of sex. When PA was adequate (in $14.4 \%$ of cases), $9.6 \%$ were users of TD and $20 \%$ of AL, whereas, when PA was inadequate, the levels of users practically did not change in percentage (TD=10.5 $\%$ and $\mathrm{AL}=20.4 \%$ ). Thus, it is concluded that the LS of university students is not suitable for health and risk reduction. There was no interference from regular and adequate physical activity in relation to the use of hazardous substances.
\end{abstract}

Keywords: Lifestyle; Hazardous substances; Physical activity.

\section{Resumen}

El objetivo de este estudio fue analizar el estilo de vida (EV) de estudiantes universitarios que consumen sustancias peligrosas y asociarlo a la actividad física diaria. Para ello, se investigaron 1405 estudiantes universitarios de la Universidad Estatal de Paraná campus de Paranavaí en Paraná en los que 330 (23,4\%) fueron identificados como consumidores de tabaco y / o drogas (TD) y 401 (28,5\%) de alcohol (AL). Además, alrededor del $85 \%$ de los estudiantes universitarios no tenían hábitos saludables de actividad física (AF). El EV se midió mediante el cuestionario "Estilo de Vida Fantástico". Así, se observó que la LS de los estudiantes universitarios que consumen sustancias nocivas, en promedio, se consideró inadecuada (TD = 58,7 $\pm 10,9 ; \mathrm{AL}=59,6 \pm 11,0$, adecuada cuando LS $\geq 70$ pts) independientemente del sexo. Cuando la AF fue adecuada (en el 14,4\% de los casos), el 9,6\% fueron usuarios 
de TD y el $20 \%$ de $\mathrm{AL}$, mientras que, cuando la $\mathrm{AF}$ fue inadecuada, los niveles de usuarios prácticamente no variaron en porcentaje $(\mathrm{TD}=10,5 \%$ y $\mathrm{AL}=20,4 \%)$. Así, se concluye que la LS de los estudiantes universitarios no es adecuada para la salud y la reducción de riesgos. No hubo interferencia de la actividad física regular y adecuada en relación con el uso de sustancias peligrosas.

Palabras clave: Estilo de vida; Sustancias peligrosas; Actividad física.

\section{Introdução}

O conhecimento pré-existente e as atitudes adquiridas através dos relacionamentos interpessoais do ser humano estão diretamente ligados a aquisição de hábitos considerados adequados e inadequados para a saúde desde a prática regular de atividade física até ao consumo de substâncias nocivas à saúde (Costa, Ribeiro \& Santos, 2019; Rodrigues \& Reis, 2020). São inúmeros os fatores que influenciam estas atitudes como: fatores socioculturais e biológicos, oportunidades, autoestima e experiências anteriores (Añez, Reis \& Petroski, 2008), dessa forma caracterizando o estilo de vida. Sendo assim, o estilo de vida (EV) pode ser definido como um padrão de hábitos que podem ser modificáveis, e, dependendo das escolhas do indivíduo, podem afetar profundamente sua saúde (Bührer, Tomiyoshi, Furtado \& Nishida, 2019).

Há várias discussões relacionadas aos benefícios dos hábitos saudáveis para a saúde, dentre elas está a afirmação de que a adoção de hábitos saudáveis na infância aumentará chance de que essa pessoa chegará na vida adulta com o estilo de vida mais seguro e saudável (de Oliveira, Oliveira, Ferreira \& Machado, 2018). Porém, o processo de transição dos adolescentes da Educação Básica para o Ensino Superior, pode influenciar na adesão de hábitos nocivos à saúde que consequentemente serão adotados na vida adulta. Neste processo, muitos jovens, devido a adaptação do modo de vida, acabam se relacionando com diferentes tipos de pessoas, formando novas amizades e mudando drasticamente sua rotina diária, sendo que dentre estas modificações pode ocorrer a necessidade de trabalhar e a mudança de residência (Luna et al., 2018), de outra forma até mesmo adotando hábitos de risco como consumo de álcool, drogas, tabaco, entre outros (Barbosa, Asfora \& Moura, 2020; Flesch, Houvèssou, Munhoz \& Fassa, 2020; E. S. de Oliveira et al., 2020; Pinho, Souza, Portugal \& Siqueira, 2020).

O consumo de drogas, tabaco e álcool é um fenômeno que tem grandes possibilidades de acarretar problemas psicossociais que podem ser apresentados em médio e longo prazo afetando, de alguma forma toda a sociedade (Elsy, Clara, Lester, Nataly \& Alejandra, 2018). Da mesma maneira que o uso de drogas ilícitas vem sendo um problema em países desenvolvidos, este desafio também pode ser enfrentado no ambiente universitário, uma vez que o universitário está com uma maior vulnerabilidade para a adesão de comportamentos nocivos à saúde (Souza et al., 2018).

Apesar de existirem diferentes determinantes relacionados às causas do uso de drogas, a educação é uma das principais quando o assunto é a prevenção. Em uma pesquisa realizada em escolas do município de São Paulo, destacou-se que um dos grandes problemas para a implementação da educação sobre drogas na escola é a falta de formação e a capacitação de professores (Pereira, Paes \& Sanchez, 2016). A Educação Física Escolar pode ser um contribuinte positivo no combate ao uso de substâncias nocivas como as drogas, uso de álcool, tabaco, pois tem hoje um papel muito importante na formação do cidadão ao longo da vida escolar, formando o aluno com capacidades suficientes para identificar atividades físicas e ações que promoverão sua saúde e bem-estar, assim como prevenção contra o uso de drogas lícitas e ilícitas. Por outro lado, em outro momento como estudante, em nível universitário, é possível que o estilo de vida não seja adequado à saúde, e ainda, o uso de substâncias nocivas ao organismo possa estar mais presente e evidenciado no cotidiano do estudante (Betti \& Zuliani, 2002). Acredita-se que a adoção de comportamentos mais seguros e saudáveis possa ser contribuinte para a modificação do modo de vida, em outros aspectos, para melhorar a saúde e segurança, já o contrário também ocorre. Sendo assim, o objetivo foi analisar o estilo de vida de universitários usuários de substâncias nocivas e associá-la com a atividade física do cotidiano. 


\section{Metodologia}

Esta pesquisa teve caráter descritivo e exploratório (Thomas, Nelson \& Silverman, 2012) com abordagem qualitativa (Pereira et al., 2018) na qual coleta e análise informações de uma população específicas sendo essas informações ainda pouco conhecidas. O estudo teve aprovação pelo Comitê de Ética da Universidade Estadual de Maringá CAAE 61069916.8.0000.0104 e parecer número 2.093.464.

A população foi composta por todos os universitários regularmente matriculados em uma instituição de ensino superior pública do município de Paranavaí-PR que estiveram presentes em sala de aula e se propuseram a ser voluntários em participar da pesquisa $(\mathrm{n}=1405)$.

O questionário padronizado Estilo de Vida Fantástico (EVF) (Añez et al., 2008) foi utilizado para identificar o estilo de vida (EV). O questionário é composto por 23 questões com respostas que variam de 0 a 4 pontos (pts.) e 2 questões dicotômicas com os valores 0 e 4 pts. Contempla nove domínios do estilo de vida formando um anagrama "FANASTIC" ( $\mathrm{F}=$ família; $\mathrm{AF}=$ =atividade física; $\mathrm{N}=$ nutrição; $\mathrm{TD}=$ tabaco e drogas; $\mathrm{AL}=a ́$ lcool; $\mathrm{S}=$ =sexo, comportamento preventivo e estresse; T=tipo de personalidade; $\mathrm{I}=$ introspecção; $\mathrm{C}=$ carreira). Após respondidas as questões somam-se os escores e observa-se o resultado podendo variar de zero a 100 pts. Quanto mais alto o escore melhor, mais saudável e seguro é o EV.

A aplicação do questionário foi realizada em sala de aula com a presença de todos os estudantes presentes e que aceitaram participar da pesquisa. O instrumento questionário de EV Fantástico é autoexplicativo, porém os pesquisadores estavam presentes em sala para orientarem inicialmente no preenchimento do formulário. Para análise estratificada foi verificado resultado proporcional em cada domínio e por sexo.

Os escores foram considerados a partir da dicotomização dos resultados sendo que valores 0,1 e 2 foram considerados como “inadequados” (EV não saudável e menos seguro) e respostas 3 e 4 como "adequadas” (EV saudável e mais seguro).

Para a análise dos domínios atividade física (AF), álcool (AL) e tabaco e drogas (TD) utilizou-se do mesmo critério adotado para identificação do EV geral. Quando $70 \%$ da pontuação máxima era atingida por cada domínio (AF=8pts; $\mathrm{AL}=12$ pts; $\mathrm{TD}=16 \mathrm{pts}$ ) era considerado EV adequado, mais saudável e seguro nos domínios.

Os dados foram analisados através da estatística descritiva com valores expressos em percentual, média e desvio padrão geral, por domínio do estilo de vida e por sexo. Testes paramétricos (“t” e ANOVA) foram usados para verificar diferenças entre escores no EV dos estudantes. Estatística não paramétrica a partir do teste Qui-quadrado foi usada para análise dos resultados dicotomizados. Em todos os testes estatísticos o grau de confiança foi de $95 \%$.

\section{Resultados}

Tendo como o objetivo deste estudo analisar o estilo de vida de universitários usuários de substâncias nocivas e associá-la com a atividade física do cotidiano. De acordo com os dados expressos na Tabela 1 observa-se que EV dos universitários obtiveram em média escore inadequado $(65,4 \pm 10,6$ pts.). Vale ressaltar que este escore foi obtido a partir da soma de todos os valores obtidos em cada domínio abordado no questionário, sendo eles: família; atividade física; nutrição; tabaco e drogas; álcool; sexo, comportamento preventivo e estresse; tipo de personalidade; introspecção; carreira.

Ao observar os usuários de substâncias nocivas, TD e AL, isoladamente percebeu-se que o EV em média decrescia $(\mathrm{TD}=58,7 \pm 10,9 ; \mathrm{AL}=59,6 \pm 11)$ sem apresentar diferença significante entre sexos (Tabela 1). De outra forma observa-se que o uso de TD e AL é prevalente em 23,4\% e 28,5 respectivamente, além disso, 11,2\% dos casos relataram fazer uso simultâneo de TD e AL, sendo um fator que afeta negativamente o estilo de vida destes usuários. 
Tabela 1 - Estilo de vida dos universitários e universitários autodeclarados usuários de álcool (AL), tabaco e drogas (TD). Valores em média e desvio padrão, \% casos e significância $\mathrm{P} \leq 0,05$ :

\begin{tabular}{lcccc}
\hline & $\mathbf{n}(\boldsymbol{\%})$ & Universitários & Masculino & Feminino \\
\hline Universitários & $1405(100)$ & $65,4 \pm 10,6$ & $65,0 \pm 11,1$ & $65,0 \pm 11,1$ \\
TD & $330(23,4)$ & $58,7 \pm 10,9^{\mathrm{a}}$ & $57,9 \pm 11,5$ & $58,6 \pm 10,4$ \\
AL & $401(28,5)$ & $59,6 \pm 11^{\mathrm{b}}$ & $59,8 \pm 11$ & $59,5 \pm 11$ \\
TD+AL & $158(11,2)$ & $52,8 \pm 11,1^{\mathrm{c}}$ & & \\
\hline
\end{tabular}

Nota: EV: Estilo de Vida; TD: Tabaco e drogas; AL: álcool;

${ }^{a}$ Diferença estatística em relação à $\mathrm{AL}$ e TD+AL $(\mathrm{P} \leq 0,05)$;

${ }^{\mathrm{b}}$ Diferença estatística em relação à $\mathrm{TD}$ e $\mathrm{TD}+\mathrm{AL}(\mathrm{P} \leq 0,05)$;

${ }^{c}$ Diferença estatística em relação à $\mathrm{TD}$ e $\mathrm{AL}(\mathrm{P} \leq 0,05)$;

Fonte: Autores.

A Tabela 2 apresenta os valores em média da pontuação alcançada pelos universitários nos domínios TD, AL e AF. Considerando que os escores máximos possíveis nesses domínios são respectivamente 16 pts., 12 pts. e 8 pts., neste caso, para o EV ser considerado adequado a pontuação para cada domínio deveria atingir pelo menos $70 \%$ dos pontos por domínio. Sendo que para atingir um escore adequado no domínio Tabaco e drogas o sujeito deveria atingir uma pontuação mínima de 12 pontos, no domínio Álcool um escore mínimo de 9 pontos e no escore do domínio Atividade física o indivíduo deveria alcançar pelo menos 6 pontos.

Tabela 2 - Comparação entre médias do estilo de vida de universitários relativas ao domínio TD e AL e relação com atividade física. Valores em média, desvio padrão e \% de casos:

\begin{tabular}{lccc}
\hline & Usuários & n $(\boldsymbol{\%})$ & EV \\
\hline AF Adequada & TD & $202(14,4)$ & $14 \pm 1,76$ \\
& AL & $18(9,6)$ & $10 \pm 2,98$ \\
AF Inadequada & & $38(20)$ & \\
\cline { 2 - 4 } & TD & $1203(85,6)$ & $13,8 \pm 2,16$ \\
& AL & $118(10,5)$ & $10,1 \pm 2,71$ \\
\hline
\end{tabular}

Nota: \% uso: percentual dos casos que relataram serem usuários de TD ou AL; AF: Atividade física; n uso: número de casos usuários de TD ou AL; AL: Álcool (adequado quando >9pts); TD: Tabaco e drogas (adequado quando >12pts); Fonte: Autores.

Ao analisar o nível de atividade física dos usuários (Tabela 2), verificou-se que $202(14,4 \%)$ pessoas apresentaram um nível de atividade física adequado. Desses indivíduos, 9,6\% relataram serem usuários de TD e $20 \%$ de AL. Neste caso a maior parte dos universitários apresentaram um nível de atividade física inadequado $(85,6 \%)$. Embora a amostra considerada com o nível de atividade física inadequada tenha sido superior os níveis de usuários praticamente não se alteravam percentualmente $(\mathrm{TD}=10,5 \%$ e $\mathrm{AL}=20,4 \%)$.

\section{Discussão}

De forma geral os universitários, tanto homens quanto mulheres, regularmente matriculados em uma instituição de ensino superior pública do município de Paranavaí-PR apresentaram, em média, um EV inadequado.

A fase de transição dos jovens para o meio universitário é marcada por várias alterações na sua rotina de atividades, bem como nas crenças e valores culturais, familiares, religiosos, entre outros relacionados ao processo de educação. Esses 
fatores podem alterar substancialmente as atitudes no dia a dia, podendo refletir de forma positiva ou negativa no seu estilo de vida (Belem, Camargo \& Both, 2019; Silva, Quadros, Gordia \& Petroski, 2011).

Neste estudo tanto os homens como as mulheres apresentaram, em média, um EV inadequado, fato esse que percentualmente piora à medida em que há presença do consumo de álcool, tabaco, drogas, ou mesmo o poli consumo dessas substâncias nocivas. Além disso, diferente do que defendem alguns autores, a aquisição de hábitos saudáveis, assim como manter-se ativo fisicamente, poderia favorecer positivamente para melhora no estilo de vida geral (Cunha et al., 2019; Esteves, Vieira, Brás, O’Hara \& Pinheiro, 2017; Nahas, Barros \& Francalassi, 2000; Neto et al., 2019), contudo, nessa pesquisa isso não ocorreu, uma vez que percentualmente, mesmo os universitários que apresentaram a $\mathrm{AF}$ como adequada demonstram um percentual de consumo de drogas próximo àqueles que apresentaram $\mathrm{AF}$ inadequada. De forma diferente, em um estudo realizado com dados pesquisa sobre comportamento em saúde no ensino superior de Utah, Estados Unidos, foi demonstrado que o afastamento dos jovens da prática de atividades físicas diárias esteve associado ao uso do tabaco (Eaves et al., 2017).

Sabendo que o ambiente universitário pode influenciar de forma negativa os alunos a adquirirem hábitos inadequados, as experiências anteriores do aluno em seu período de adolescência também podem influenciar na continuidade de hábitos na vida adulta (Gallahue, Ozmun \& Goodway, 2013), nesse caso, se for positivo se tornando favorável ao adequado e saudável estilo de vida. Um estudo realizado com adolescentes do sul brasileiro, apresentou resultados semelhantes a este estudo em relação a associação do consumo de bebidas alcoólicas e o tabagismo com a atividade física, na qual, também, a atividade física não teve influência no consumo dessas substâncias nocivas à saúde (Lima \& Silva, 2018).

Em relação a adesão às atividades físicas pelos universitários, pode-se destacar a procura por melhoras na aptidão física, diminuição do estresse, diversão, prevenção de doenças, aumento da autoestima, aquisição de hábitos saudáveis, definição muscular, socialização, perda de peso e reabilitações. Já, em relação a não participação em atividades físicas estão associadas as causas como indisponibilidade de horário, localização inoportuna de ambientes para prática, custo, inaptidão para tipos de atividades, falta de interesse, falta de identificação com os outros participantes e com a forma de organização das atividades (Esteves et al., 2017). Esses aspectos ressaltam como a ausência de práticas regulares da atividade física também podem contribuir para afetar negativamente aspectos sociais e mentais do indivíduo além dos físicos.

Em uma pesquisa realizada com 1369 estudantes de uma universidade do norte da Inglaterra os autores buscaram explicar os motivos pelos quais os universitários faziam o consumo compulsivo de bebidas alcoólicas e o tabagismo. Para o fator bebida a justificativa da maioria dos entrevistados era de que o consumo de bebidas alcoólicas é divertido e é uma boa opção para fazer novos amigos, porém também houve relatos de estudantes de que o consumo de bebidas alcoólicas poderia gerar um impacto negativo no seu rendimento. Quando questionados sobre como os amigos e os pais reagiriam sobre este consumo, foi relatado que os amigos aprovariam e os pais desaprovariam esta ideia. Portanto, o estudo apresenta motivos pelos quais os alunos começariam o obter este hábito destacando-se entre eles o ato de ver outras pessoas fazendo o consumo excessivo de álcool (Epton et al., 2014). Já o que levou a maioria dos alunos ao tabagismo, segundo Epton et al. (2014) foi a busca pelo alívio do estresse diário e a socialização, algo que se devidamente praticada, as atividades físicas teriam efeitos muito mais saudáveis.

Embora o consumo de bebidas alcoólicas seja comum no ambiente universitário e pela maioria dos jovens, este consumo está associado a perda de habilidades motoras, talvez daí uma certa restrição dos consumidores com as atividades motoras, em especial que requerem habilidades mais específicas como esportes. Um estudo realizado nas Escolas da Universidade Gimbernat-Cantabria na Espanha, demonstrou que os estudantes que bebiam tiveram um desempenho pior nos testes de atenção e flexibilidade mental do que os estudantes que não faziam o consumo de bebidas alcoólicas. Ao ser ajustado de acordo com a idade, sexo, histórico acadêmico, idade inicial de consumo de álcool, consumo de cannabis, nível de atividade física e outras variáveis modificadoras ou fatores de confusão como a hora do dia, o pesquisador e o dia da semana em que os 
testes foram realizados, a associação entre teste e o consumo de bebidas alcoólicas se manteve, reforçando a ideia de que maus hábitos podem levar prejuízos à saúde (Salas-Gomez et al., 2016).

O ganho de peso nos calouros, do sexo masculino, do primeiro semestre de uma universidade dos Estados Unidos esteve associado ao consumo de álcool entre os estudantes, já, entre as mulheres o ganho de peso esteve relacionado com a prática de atividades físicas (Bodenlos, Gengarelly \& Smith, 2015).

Em relação ao hábito de consumir bebida alcoólica tanto regularmente quanto de forma excessiva, bem como, para o consumo de maconha, os homens apresentaram piores hábitos em relação as mulheres, porém em relação ao consumo de outras drogas do tipo recreacionais as mulheres apresentaram hábitos mais inadequados em relação aos homens (Tran et al., 2017). Em quaisquer situações, o comprometimento causado pelo uso das substâncias nocivas contribuía para afetar negativamente outros aspectos da vida cotidiana dos indivíduos

Um levantamento realizado pela Secretaria Nacional de Políticas Sobre Drogas, em 2010, apontou que 48,7\% de universitários de redes públicas e privadas de todas as capitais do Brasil já fizeram ou fazem a utilização de drogas ilícitas. Além disso, não se contabilizou o uso do álcool ou algum produto derivado do tabaco. O mesmo estudo indica que 86,2\% e 46,7\%, dos avaliados, também já fizeram uso de álcool e produtos do tabaco respectivamente (Nicastri, Oliveira, Wagner \& Andrade, 2010). Neste estudo o uso de TD e AL variou entre cerca de 23,4\% e 28,5\% dos relatos com universitários.

Um estudo realizado em universitários da área de ciências da saúde em Santo André, município do ABC paulista, destacou que $27,9 \%$ da amostra fazia o poli consumo de drogas, combinando: "álcool + Cannabis; álcool + medicamentos prescritos; álcool + tabaco + Cannabis; tabaco + Cannabis; e tabaco + medicamentos prescritos" (Nóbrega et al., 2012). Neste caso $11,2 \%$ dos universitários apresentaram fazer o poli consumo entre TD+AL. O estudo ainda apresenta algumas justificativas que os usuários diziam ao serem questionados como motivadores do poli consumo: relaxamento, perda de inibições e manter-se acordado. Independente de se obter sensação momentânea de satisfação e prazer, as consequências para o indivíduo e seu meio social desse tipo de comportamento, se habitual no estilo de vida, poderiam acarretar sérios problemas à saúde.

\section{Conclusão}

Estudantes universitários apresentam estilo de vida instável e preocupante quando relacionado à prevenção de doenças e riscos no dia a dia devido ao predominante estilo de vida inadequado. Essa condição é elevada independente do sexo a partir da adoção no estilo de vida de uso de substâncias conhecidas como nocivas ao organismo como álcool, tabaco e drogas. Por fim, em condições na qual o poli uso dessas substâncias ocorriam o estilo de vida geral era ainda mais depreciado. Essa condição não se alterava quando universitários eram identificados como adequados na atividade física geral.

Sugere-se programas de apoio, orientação e conscientização aos estudantes universitários para possibilitar condições preventivas e quando necessárias terapêuticas no combate às substâncias nocivas ao organismo ao longo da vida acadêmica. A universidade deveria também cumprir um papel de educação para saúde e para a vida.

Embora os dados deste estudo serem relevantes para a elaboração de políticas públicas que diminuam estes comportamentos nocivos à saúde no ambiente universitário, destaca-se a importância de desenvolvimento de estudos que investigue quais são as causas da adoção destes hábitos inadequados.

\section{Referências}

Añez, C., Reis, R. \& Petroski, E. (2008). Artigo Original Versão Brasileira do Questionário “ Estilo de Vida Fantástico ": Arquivos Brasileiros de Cardiologia, 91 (2), s. 102-109.

Barbosa, L. N. F., Asfora, G. C. A. \& Moura, M. C. de (2020). Ansiedade e depressão e uso de substâncias psicoativas em jovens universitários. SMAD 
Revista Eletrônica Saúde Mental Álcool e Drogas, 16 (1), s. 1-8.

Belem, I. C., Camargo, D. A. D. \& Both, J. (2019). Diferent lifestyle behaviors of college students from the Physical Education course. Revista de Ciencias del Ejercicio y la Salud, 17 (2), s. 1-14.

Betti, M. \& Zuliani, L. R. (2002). Educação Física Escolar: uma proposta de diretrizes pedagógicas. Revista Mackenzie de Educação Física e Esporte, 1 (1), s. 73-81.

Bodenlos, J. S., Gengarelly, K. \& Smith, R. (2015). Gender differences in freshmen weight gain. Eating Behaviors, 19, s. 1-4.

Bührer, B. E., Tomiyoshi, A. C., Furtado, M. D. \& Nishida, F. S. (2019). Análise da Qualidade e Estilo de Vida entre Acadêmicos de Medicina de uma Instituição do Norte do Paraná. Revista Brasileira de Educação Médica, 43 (1), s. 39-46.

Costa, R. A. de O. S., Ribeiro, J. L. O. A. \& Santos, M. R. dos (2019). A Contribuição Da Educação Infantil Para a Formação De Bons Hábitos Alimentares Na Criança De 0 a 6 Anos. Revista de Ciências Humanas - UNIPLAN, 1 (1), s. 32-32.

Cunha, N. et al. (2019). Relação entre género ao nível da motivação para prática da atividades desportivas. Mediações - Revista OnLine da Escola Superior de Educação do Instituto Politécnico de Setúbal, 7 (1), s. 151-162.

Eaves, E. R. et al. (2017). Demographic trends in Utah college students' vigorous physical activity, 2003-2007. American Journal of Health Behavior, 41 (4), s. $437-445$.

Elsy, L. G., Clara, V. G. N., Lester, W. V., Nataly, L. C. G. \& Alejandra, C. C. M. (2018). Caracterización del consumo de sustancias lícitas e ilícitas en los estudiantes de Medicina de UNIANDES. Rev UNIANDES Cienc Salud, 1 (1), s. 28-42.

Epton, T. et al. (2014). Development of theory-based health messages: Three-phase programme of formative research. Health Promotion International, 30 (3), s. $756-768$

Esteves, D., Vieira, S., Brás, R., O’Hara, K. \& Pinheiro, P. (2017). Nível de atividade física e hábitos de vida saudável de universitários portugueses. Revista Iberoamericana de Psicología del Ejercicio y el Deporte, 12 (2), s. 661-270.

Flesch, B. D., Houvèssou, G. M., Munhoz, T. N. \& Fassa, A. G. (2020). Major depressive episode among university students in Southern Brazil. Revista de Saúde Pública, 54, s. 11.

Gallahue, D. L., Ozmun, J. C. \& Goodway, J. D. (2013). Compreendendo o Desenvolvimento Motor: bebês, crianças, adolescentes e adultos. AAMGH (7. udg.). Porto Alegre: AMGH.

Lima, T. R. \& Silva, D. A. S. (2018). Prevalence of physical activity among adolescents in southern Brazil. Journal of Bodywork and Movement Therapies, 22 (1), s. 57-63.

Luna, A. A. et al. (2018). Caracterização do Estilo de Vida de Universitários do Ensino Superior a Distância. Journal of Health Sciences, 20 (1), s. 40.

Nahas, M., Barros, M. V. G. de \& Francalassi, V. (2000). O Pentáculo do bem-estar - Base conceitual para avaliação do estilo de vida de indivíduos ou grupos. Revista Brasileira de Atividade Física \& Saúde, 5 (2), s. 48-59.

Neto, A. P. V. et al. (2019). Evaluation of body satisfaction ad use of anorexic and anabolic steroids substances in university students. Revista Interdisciplinar de Estudos Experimentais, 10 (Único), s. 15-23.

Nicastri, S., Oliveira, L. G. de, Wagner, G. A. \& Andrade, A. G. de (2010). Prevalência e padrão de uso de tabaco e outras drogas (exceto álcool): estimativa de abuso e dependência. I: A. G. de Andrade, P. do C. A. V. Duarte \& L. G. de Oliveira (Red.), I levantamento nacional sobre o uso de álcool, tabaco e outras drogas entre universitários daas 27 capitais brasileiras (1. udg., s. 53-81). Brasília.

Nóbrega, M. P. S. de S. et al. (2012). Policonsumo simultâneo de drogas ente estudantes de graduação da área de Ciencias da Saúde de uma universidade: implicações de gênero, sociai e legais, Santo André - Brasil. Texto \& Contexto - Enfermagem, 21(spe), 25-33

Oliveira, E. S. et al. (2020). Stress and health risk behaviors among university students. Revista Brasileira de Enfermagem, 73 (1), s. 1-8.

Oliveira, F. A., Oliveira, M. L. de, Ferreira, A. R. O. \& Machado, M. F. (2018). The role of healthy food in the child development process and the teachre's responsiblity in the healthy habit formation inssue. Revista de Humanidades, Tecnologia e Cultura, 08 (1-17).

Pereira, A. P. D., Paes, Â. T. \& Sanchez, Z. M. (2016). Factors associated with the implementation of programs for drug abuse prevention in schools. Revista de Saúde Pública, 50 (0), s. 1-10.

Pereira, A. S., Shitsuka, D. M., Parreira, F. J., Shitsuka, R. (2018). Metodologia da Pesquisa Cêntífica. UFSM.

Pinho, M. C., Souza, R. C. F. de, Portugal, F. B. \& Siqueira, M. M. de (2020). Uso de álcool e tabaco entre universitários de Terapia Ocupacional de uma universidade pública. SMAD Revista Eletrônica Saúde Mental Álcool e Drogas (Edição em Português), 16 (1), s. 1-12.

Rodrigues, C. I. R. D. \& Reis, A. (2020). Do comer bem ao crescimento saudável. Rev UIIPS, 8 (1), s. 68-79.

Salas-Gomez, D. et al. (2016). Binge drinking in young university students is associated with alterations in executive functions related to their starting age. PLoS ONE, 11 (11), s. 1-12.

Silva, D. A. S., Quadros, T. M. B. de, Gordia, A. P. \& Petroski, E. L. (2011). Associação do sobrepeso com variáveis sócio-demográficas e estilo de vida em universitários. Ciência \& Saúde 16 (1), s. 4473-4480.

Souza, J. et al. (2018). Consumo de drogas e conhecimento sobre suas consequências entre estudantes de graduação em Enfermagem. Texto \& Contexto - 
Research, Society and Development, v. 10, n. 3, e35710313427, 2021

(CC BY 4.0) | ISSN 2525-3409 | DOI: http://dx.doi.org/10.33448/rsd-v10i3.13427

Enfermagem, 27(2), 1-10

Thomas, J. R., Nelson, J. K. \& Silverman, S. J. (2012). Métodos de Pesquisa em Atividiade Física. Artmed (6a ed.), Artmed.

Tran, A. et al. (2017). Health assessment of French university students and risk factors associated with mental health disorders. PLoS ONE, 12 (11), 1-18. 